\title{
Neoliberalismo, regulación ad-hoc de suelo y gentrificación: el historial de la renovación urbana del sector Santa Isabel, Santiago ${ }^{1}$
}

\author{
Ernesto López², Daniel Meza ${ }^{3}$ e Ivo Gasic ${ }^{4}$
}

\begin{abstract}
RESUMEN
La zona de Avenida Santa Isabel en la comuna de Santiago es emblemática en Chile por contener una de las mayores intensificaciones de desarrollo inmobiliario en altura de la década del 2000, con altos niveles de absorción privada de renta de suelo y desplazamiento de residentes locales originales. Este artículo documenta en detalle: 1) la adopción de un esquema municipal pro empresarial que desde 1985 orienta sus instrumentos hacia la activación del mercado inmobiliario y la amplificación de ganancia privada por mayor constructibilidad de suelo, 2) la disparidad observada entre niveles de captura de renta de suelo por desarrolladores y residentes locales y 3 ) en relación con lo anterior, el potencial "desplazamiento exclusionario" para la mitad de los actuales propietarios-residentes originales de la zona, entendiéndose como un insuficiente poder de compra de vivienda de reemplazo según el monto recibido por venta de su predio.
\end{abstract}

Palabras clave: Renovación urbana, gentrificación, brecha de renta, desplazamiento exclusionario.

\begin{abstract}
During the 2000s the sector of Avenida Santa Isabel, located within the municipality of Santiago in Santiago, Chile, was emblematic in Chile as it experiences one of the greatest increases in high-rise real estate development, with high rates of both private land purchases and displacement of original residents. This article documents in detail: 1) the evolution of a pro-business public-sector scheme, started in 1985, aimed at stimulating real estate market conditions and increasing private profit through increasing the constructible surface area; 2) the observed disparity of a reduced group of construction an/or real estate companies; and 3) in relation to the above, the potential "exclusionary displacement" experienced by half of the local owner-residents who have insufficient purchasing power for replacement housing based on the price received from the sale of their land.
\end{abstract}

Key words: Urban renewal, gentrification, rent gap, exclusionary displacement.

1 Proyecto FONDECYT No 11100337. Artículo recibido el 30 de octubre de 2013, aceptado el 11 de marzo de 2014 y corregido el 4 de junio de 2014.

2 Departamento de Urbanismo, Universidad de Chile (Chile). E-mail: elopez@uchilefau.cl
3 Universidad Federal de Río de Janeiro (Brasil). E-mail: danielmezacorvalan@gmail.com

4 Núcleo de Estudios Espacio y Capital, Departamento de Geografía, Universidad Alberto Hurtado (Chile). E-mail: ivo.humano@gmail.com 
Las principales ciudades de América Latina en las últimas décadas han experimentado procesos de reestructuración urbana en sus zonas centrales. Las estrategias generalmente pasan por asociaciones e instrumentos de promoción público-privados, que las administraciones municipales urbanas utilizan para consolidar la expansión de una lógica de mercantilización del espacio urbano como motor para procesos de reestructuración urbana física y social. La ciudad de Santiago de Chile ha sido documentada como ejemplo de una política urbana de reconfiguración de suelo central, mediante proyectos detonantes (Valenzuela, 2003) y subsidio al mercado de vivienda (Rojas, 2004; Arriagada et al., 2007). Más recientemente, Cattaneo (2011) ha expuesto el funcionamiento de sociedades de capital mixto global-local en este lugar, en un contexto institucionalizado de financiarización económica e incentivos a la elusión tributaria con el fin de lograr mayor capitalización corporativa a través de proyectos en altura y gran escala en la ciudad, dinámica crecientemente instalada en áreas centrales. Por su parte López Morales (2013b) observa que los retornos crecientes de la inversión inmobiliaria, capturados por un número limitado de empresas (no más de cuatro inmobiliarias que concentran casi el $50 \%$ de los edificios construidos en la zona Santa Isabel en ese período) provienen en gran medida de renta de suelo y no de una mayor productividad de la inversión en construcción.

En Buenos Aires no solo figuran Puerto Madero como una megaurbanización de servicios y vivienda orientada a nichos socioeconómicos altos, en suelo previamente deteriorado de alta centralidad, sino que toda la zona sur central presenta signos de reestructuración mercantil de proyectos de rehabilitación turística y transformación de inmuebles (Herzer, 2008). El caso de Ciudad de México ha sido descrito por Delgadillo (2008) como de alta incidencia de políticas públicas en la reconfiguración de ventajas ofrecidas al mercado en suelo central entre otras medidas, a través del Bando 2, y orientaciones públicas agresivas de despeje de espacio público de la venta informal de productos en zonas históricas centrales de alto consumo turístico. Por su parte, Janoschka et al. (2014) han compilado y comentado las distintas experiencias observadas en el subcontinente hasta el año
2012, pero aún quedan preguntas pendientes acerca del funcionamiento de cada contexto específico donde confluyen las transformaciones sociodemográficas, cambios culturales y dominios de clase sobre los espacios urbanos, políticas estatales de suelo, y las distintas dinámicas de mercado inmobiliario. El presente dossier especial de Norte Grande apunta en parte a ese objetivo.

En Chile, desde mediados de la década de 1980 y con el fin de recuperar espacios de alta centralidad y revertir los efectos de al menos tres décadas previas de despoblamiento por migración centrífuga hacia la periferia con descenso del nivel socioeconómico promedio de los hogares residentes centrales, el Estado comenzó un proceso de configuración de líneas estratégicas públicas y municipales de renovación urbana a materializarse por agentes privados, a saber: a) implementación de agencias privado-municipales para atraer y anclar al centro y pericentro nuevos desarrollos inmobiliarios; b) aplicación de un subsidio para la adquisición de viviendas económicas (inferiores a $140 \mathrm{~m}^{2}$ ) en un área definida como Zona de Renovación Urbana; y c) orientación de los Instrumentos de Planificación Territorial (IPT) para establecer condiciones que posibiliten el desarrollo inmobiliario en las diferentes coyunturas, redistribuyendo complementariamente otros usos del suelo funcional a la densificación habitacional.

Si bien en la actualidad el alcance de esta estrategia pública se extiende a las once comunas del centro y pericentro de Santiago ${ }^{5}$, inicialmente la comuna de Santiago, cuyo núcleo es el sector fundacional de la ciudad, fue pionera en Chile (desde 1985) en definir una estrategia de renovación local articulada con incentivos de subsidio de Estado central y con objetivos trazados a largo plazo (Valenzuela, 2003). Se estima que desde 2007 en adelante, el volumen construido y de unidades habitacionales producidas por el modelo privado de renovación urbana en altura en

\footnotetext{
5 Comenzando desde el "core" metropolitano y en sentido opuestos a los punteros del reloj: Santiago Centro, Recoleta, Independencia, Renca, Quinta Normal, Estación Central, Cerrillos, Pedro Aguirre Cerda, San Miguel, San Joaquín, y Macul.
} 
comunas centrales y pericentrales del Área Metropolitana del Gran Santiago (AMGS) es mayor que el de la construcción de casas unifamiliares en la periferia. Asimismo, la participación de la comuna de Santiago en la producción privada de departamentos es mayoritaria a nivel regional, con un $53,8 \%$ de las unidades de departamentos producidos entre 2002-2011, alcanzando incluso, en el año 2011, el 46\% del total de unidades producidas en la región, tanto en departamentos como en casas unifamiliares ${ }^{6}$.

Las acciones de renovación urbana comenzadas en la comuna de Santiago Centro en 1985, y expandidas luego al pericentro durante las siguientes dos décadas, han modificado la lógica de localización residencial del desarrollo urbano en el Gran Santiago. La ciudad es cada vez más una metrópolis que se "verticaliza", a la par que mantiene procesos acentuados de expansión en baja densidad hacia comunas periferias distantes dentro del conjunto de las 52 comunas de la Región Metropolitana. Esto último se debe en gran medida a los aún bajos costos comparativos de suelo periférico (discontinuo a la mancha urbana) a absorber por desarrolladores privados de vivienda social (en Chile no existe construcción de vivienda social directamente por agencia pública) y una todavía alta demanda de espacios de condiciones ambientales "verdes" por parte del mercado residencial suburbano. Según De Mattos y Fuentes (2012) este patrón constituye un nuevo tipo de dualidad, basada en procesos simultáneos de concentración y dispersión, ambos siguiendo lógicas de consumo de suelo con altos márgenes de rentabilización.

Este artículo se focaliza en la evolución, desde la década de 1980, del sector Santa Isabel, un cuadrante de 30 hectáreas situado en el corazón de la comuna central de Santiago. Santa Isabel durante la década del 2000 experimentó una de las "verticalizaciones" más radicales en ciudades chilenas, y se configura como un caso representativo y reciente de confluencia entre gestión municipal pro empresarial, explotación inmobiliaria con

\footnotetext{
6 Según datos del Observatorio Habitacional MINVU (2011).
}

maximización de capitalización por renta de suelo, y tendencia al desplazamiento de residentes originales de bajos ingresos. El artículo plantea una narrativa ordenada cronológicamente de la siguiente forma: en la sección siguiente, se analiza el rol de la CORDESAN, una agencia mixta municipal-privada creada a fines de la década de 1980 para gestionar suelo y demanda necesaria para la activación del mercado de renovación urbana en Santiago. Se analiza luego el rol e impacto del Subsidio de Renovación Urbana, aplicado desde 1991, y el rol aún más importante de los mecanismos de regulación de la constructibilidad de suelo (superficie total construida / superficie de la parcela suelo), redefinidos en la comuna a partir de 1989, y cuya potestad la tiene el municipio para la activación de nichos de inversión con alto retorno por ganancia de renta de suelo. Finalmente, se entregan resultados de un análisis realizado para el periodo 2000-2012 referido al potencial de "desplazamiento exclusionario" que ha generado la verticalización de Santa Isabel, y donde un $50 \%$ de hogares residentes propietarios, tras vender sus parcelas de suelo, no encontrarían posibilidades económicas de acceder a la oferta de vivienda nueva dentro de la misma zona, y por ende se verían gentrificados por sujetos de mayor poder adquisitivo. Utilizamos la definición genérica de gentrificación de Eric Clark (2005), explicada en la sección correspondiente.

\section{Renovación urbana de la comuna de Santiago y sector Santa Isabel}

En Chile, la lógica subsidiaria de renovación urbana tiene su origen en la comuna de Santiago, ya que este municipio materializó directamente a inicios de la década de 1990 los primeros proyectos pilotos en los cuales se negoció con el Ministerio de Vivienda y Urbanismo (MINVU) la aplicación de 200 subsidios a la adquisición de vivienda nueva en modalidad de departamentos, a través del Programa Especial de Trabajadores. Fruto de esta gestión fue la implementación en 1991 del Subsidio de Renovación Urbana, que pasó a ser una de las herramientas del Estado para promover la acción de agentes privados inmobiliarios en las zonas centrales de las principales ciudades del país, entregando 
un subsidio de 200 UF $^{7}$ a la demanda de vivienda de tipo DFL- ${ }^{8}$ (es decir, de precio máximo hasta $2.000 \mathrm{UF}$ ), pero que luego se traspasa indirectamente a la oferta a través del aumento sostenido del precio de venta de las viviendas (Rojas, 2004).

En segundo lugar, en ese entonces fue necesario también establecer una nueva institucionalidad capaz de incorporar a agentes privados en la toma de decisión del municipio de Santiago, en una "descentralización de la gestión urbana" ${ }^{\prime \prime}$. Se diseñó para ello, y tras el terremoto de 1985, la Corporación para el Desarrollo de Santiago (CORDESAN), institución privada de capital mixto sin fines de lucro, orientada a la coordinación público-privada en la toma de decisiones, con el objetivo de catalizar el proceso de reconstrucción posterremoto y permitir la entrada del sector privado al decaído mercado inmobiliario comunal. En dicha mesa directiva, dirigida por cada alcalde de turno, se encontrarían miembros de la Cámara Chilena de la Construcción, directores de las principales empresas inmobiliarias, instituciones dedicadas a promover el desarrollo de iniciativas privadas en la comuna (por ejemplo, "Barrio Universitario de Santiago", ya en la década de 2000), entre otros. Cabe destacar que esta experiencia pionera de la CORDESAN se ha intentado replicar, con adaptaciones, para el desarrollo en otras comunas de la misma

7 La UF es una unidad de cuentas autoajustable según inflación, ampliamente utilizada en Chile. Su valor a junio 2014 es de cerca de $\$ 24.000$ pesos chilenos, equivalente a US\$ 43.

8 Decreto de Fuerza de Ley $\mathrm{N}^{\circ} 2$ proveniente del año 1959, que otorga un beneficio de exención del pago de contribuciones de bienes raíces hasta por 20 años a los compradores de nuevas "viviendas económicas" de precio bajo 2.000 UF. Dado que las contribuciones se traspasan directamente al Fondo Común Municipal, esta recaudación no se reinvierte directamente en la comuna desde donde se recolecta.

9 Se llevaron a cabo una serie de seminarios, en conjunto con la Pontificia Universidad Católica de Chile, en abril de 1990 titulados "Desarrollo Económico y Urbano en un sistema de Gestión Municipal Descentralizada" donde se difundió principalmente esa idea como forma de fortalecer los gobiernos locales. Académicos y directores técnicos ligados al municipio reconocen esa instancia decisiva en lo que sería la gestión urbana del Programa de Repoblamiento. ciudad, como Independencia y Cerrillos en el caso puntual del proyecto Ciudad Parque Bicentenario (Zunino, 2006).

La CORDESAN desde fines de los años 80 realizó funciones necesarias de compra y fusión de terrenos para desarrollo en altura, incluso saneando la situación de predios que presentaban problemas legales de tenencia y sucesiones. No hubo entonces problemas para llevar adelante prácticas desarrollistas con fuerte intervención municipal, en lo que fue la creación de: a) un Banco de Terrenos destinado a la renovación urbana; b) bolsas de demanda, agrupadas y destinadas a cada proyecto, previo a su construcción; y c) asociación con entidades de la banca privada, como el Banco del Desarrollo, que en el inicio de la CORDESAN otorgó créditos hipotecarios de hasta 25 años plazo, para financiar el monto no cubierto por el ahorro familiar y el subsidio estatal de renovación urbana (CORDESAN, 1995). Complementariamente, esta agencia municipal-privada desarrolló una serie de programas de alcance indirecto a la renovación urbana, como el Programa de Mejoramiento de Espacios Públicos, el Programa de Pintura de Fachadas (aplicado en inmuebles con valor patrimonial y cités), y remodelación de espacios públicos, entre otras acciones (Contreras, 2011).

Sin embargo, al igual que ocurrió con el Subsidio de Renovación Urbana, en la medida que la demanda por residencia en la comuna se incrementó, la injerencia de la CORDESAN perdió relevancia. Los propios agentes desarrolladores fueron integrando la labor de conseguir terrenos y atraer a la demanda, desarrollando carteras privadas de terrenos. Esto significó un incremento de la demanda individual en la adquisición de departamentos, independizando el proceso productivo de la necesidad de contar con una bolsa de demanda para construir, y relegando el accionar municipal a un rol de "proveedor" de constructibilidad de suelo mediante el ajuste de sus ordenanzas locales de construcción, lo que de hecho sería la tónica para el resto de las comunas pericentrales de Santiago a partir de la década siguiente (LópezMorales et al., 2012).

El Subsidio de Renovación Urbana fue fundamental durante los primeros años de 
desarrollo del proceso de renovación urbana. Según datos de la misma CORDESAN, el porcentaje de viviendas adquiridas con subsidio alcanzó en la comuna de Santiago el 92\% en 1994; pero ya en el año 2000 había bajado a $40 \%$, cuando la acción conjunta del municipio y gobierno central era también menos relevante en la activación del mercado de vivienda en altura. El rango de precios de la oferta de vivienda en la comuna también subió durante este mismo período, superando las 2.000 UF, que como ya se dijo, es el precio máximo de vivienda permitido por el mismo subsidio (Arriagada et al., 2007).

De manera simultánea fue generándose un proceso de corporatización de los agentes inmobiliarios implicados en la renovación urbana de Santiago. A mediados de los noventa, la incorporación de actores privados fue creciente, concentrándose buena parte de la producción en inmobiliarias como Inmobiliaria Paz Froimovich, RVC y Euro. Este proceso a su vez coincidió con la salida de desarrolladores de relativo rol social, como las cooperativas abiertas de vivienda, principalmente la empresa Habitacoop.

En la década de 2000, el tipo producto inmobiliario desarrollado no dista del producto generado en la etapa anterior de 1990 (en su gran mayoría, departamentos de menos de 70 $\mathrm{m}^{2}$, aunque progresivamente más pequeños en promedio), construyendo residencias tipo viviendas económicas en edificaciones de tamaños acotados, no obstante que el precio se había incrementado. En efecto, las 11 comunas centrales y pericentrales de Santiago, entre 2000 y 2010 , presentan un alza de $40 \%$ en los precios de las unidades residenciales, mientras los tamaños promedio de esas mismas unidades descendieron en una proporción similar.

La incorporación de los encadenamientos productivos asociados a gestión inmobiliaria y construcción dentro de la misma empresa, sumado a la acumulación de suelo mediante bancos de terrenos, por parte de las principales inmobiliarias, generó un efecto de aumento del poder de los agentes económicos por sobre los residentes propietarios locales en la determinación de los precios del suelo. En rigor, se trata de un mercado de suelo fundamentalmente monopsónico de pocos compra- dores y muchos vendedores de suelo (López Morales, 2013b). Esto se analiza más adelante en este artículo. En el lapso 2000-2010 el volumen de la construcción por proyecto no aumentó, aunque el volumen construido, la altura de la edificación, y la densidad sí lo hicieron, ello reflejado en el creciente coeficiente de constructibilidad proyectado.

Si a fines de la década de 1990, en la comuna de Santiago los proyectos construidos aprovechaban coeficientes de constructibilidad de 6, esto es, producían una superficie habitable de seis veces el tamaño del predio donde se erigen, durante la década siguiente y principalmente durante su última mitad, ese valor había subido a 9, muy por sobre el resto de las otras principales áreas metropolitanas receptoras de proyectos de renovación urbana. Por ejemplo, el coeficiente de constructibilidad promedio de los edificios producidos entre 2000 y 2010 en el sector Santa Isabel fue de 9,48, extremadamente alto si se le compara con otras zonas que también se desarrollaban en ese periodo, como la comuna de Ñuñoa con una tasa de 4,62 y la comuna de Recoleta, con un coeficiente de 5,45. En promedio los edificios construidos entre 2000 y 2010 en Santa Isabel se componen de 300 unidades de vivienda, mientras que en Ñuñoa y Recoleta los promedios son de 119 y 155 respectivamente.

A partir de la década de 2000, las inmobiliarias comenzaron a concentrar la capacidad técnica para producir de manera simultánea, ya no solo una gran cantidad de departamentos por proyecto, sino una serie de proyectos coordinados en etapas. Estas empresas además fueron progresivamente adquiriendo mejoras competitivas importantes, en la medida que empresas más pequeñas tendieron a la desaparición, por falta de financiamiento y créditos bancarios durante y con posterioridad a la crisis Asiática (1998-2002) y a la recesión global-inmobiliaria (2008-2010). Esta situación coincide con la entrada de las grandes empresas inmobiliarias a cotizar en la bolsa de valores chilena, e integrar al capital financiero global circulante, incrementando exponencialmente su capacidad económica.

La inmobiliaria Paz-Froimovich, denominada Paz Corp desde mediados de 2000, ejemplifica algunos de estos elementos. En 
2006 la empresa comenzó a cotizar en la bolsa, lo cual inmediatamente incide en un fuerte incremento de su capital ese mismo año. En el año 2013, el patrimonio de la compañía ascendía a unos 83.000 millones de pesos, siendo solo un $20 \%$ de este, perteneciente al grupo familiar y controlador Paz. Además, su mayor inversionista es la empresa norteamericana de seguros y servicios financieros Prudential Financial, quienes, a través de capitales provenientes de pensiones y seguros internacionales, compraron en 2009 el $18 \%$ de la empresa, en un claro modelo de integración y control del capital financiero global, y derivando su producción inmobiliaria de renovación urbana desde Santiago a otras ciudades de Chile, Perú y Brasil.

En la zona de Santa Isabel, desde 2006 la superficie producida por construcción nueva residencial aumentó fuertemente. Además un cierto número de empresas productoras han tendido a monopolizar gran parte de la producción. Al menos en torno al eje Santa Isabel existe una concentración de un $48 \%$ de la producción de superficie nueva construida en tan solo cuatro empresas (Euro, Paz Froimovich, ABSAL y RVC) (López Morales et al., 2013b). Estas inmobiliarias generan un producto residencial homogéneo, predominando la oferta de unidades de acotadas dimensiones, inclusive llegando a producir en un $90 \%$ tipologías de departamentos de uno y dos dormitorios, excluyendo en gran medida a grupos familiares más extensos. Asimismo, al mantener precios de venta constantes y con una tendencia de disminución del tamaño en los departamentos, ha aumentado el precio de cada metro cuadrado útil ofertado por departamento. Si entre 2001 y 2004, el precio de venta de un metro cuadrado de departamento nuevo era de $31 \mathrm{UF} / \mathrm{m}^{2}$, entre 2010 y 2012, ese valor había aumentado a 40 $\mathrm{UF} / \mathrm{m}^{2}$.

Ya hemos establecido que un factor clave en la generación de proyectos en Santiago a comienzos de la década de 1990 fue la acción de la CORDESAN, que integró gestión y ejecución de programas. Sin embargo, una vez detonada la renovación urbana, como un fenómeno generalizado en la comuna, fueron entonces las condiciones de regulación normativa del Plan Regulador Comunal (PRC) las que permitieron la expansión de este mer- cado a los niveles de intensidad y volumen producido exhibidos el día de hoy.

\section{El desplazamiento de la actividad inmobiliaria mediante zonificación de suelo}

En materia regulatoria de suelo, el municipio ha adoptado diversas posturas ajustando la regulación de acuerdo a sus objetivos de desarrollo urbano, pero también incluso lo ha hecho en relación con contextos anticíclicos. De esa forma, los ajustes al Plan Regulador Comunal de Santiago posterior a la Crisis Asiática en 2002 se caracterizaron por encauzar la consolidación de procesos de renovación intensos, limitando altura, coeficientes de constructibilidad y/o estableciendo zonas e inmuebles de conservación por interés patrimonial en espacios específicos, como el histórico barrio Yungay, al norponiente de la comuna. Esto ha permitido, entre otras cosas, contener el descontento vecinal de residentes de esos sectores. Sin embargo, con ello se ha ido desplazando el mercado de edificación en altura hacia otras zonas socialmente menos conflictivas y con potencial de renovación en la comuna. En entrevista con funcionario municipal, se reconoce que:

"[Somos nosotros quienes] vamos despla-
zando el accionar del sector inmobiliario,
porque donde tiene menos restricciones
es para donde ellos se van a ir [... Así, la
modificación de nuestro Plan Regulador]
se fue haciendo por sectores, y sobre todo
donde había mayor presión [social], por-
que el sector inmobiliario estaba crecien-
do demasiado en las alturas en esa zona"
(entrevista realizada a arquitecta del De-
partamento de Urbanismo del Municipio
de Santiago, abril 2012).

Cuando las regulaciones municipales de suelo se orientan a la contención del proceso de renovación urbana y su desplazamiento desde zonas a "proteger", con relocalización en otras zonas dentro de la comuna, ello no implica necesariamente que el municipio logre ese objetivo de evitar situaciones críticas de desmejoramiento de las áreas residenciales consolidadas del centro. El mismo relato municipal afirma, enfáticamente, que el accionar del municipio muchas veces se 
realiza ex post, vale decir, después de que la actividad inmobiliaria en altura ya ha penetrado y fracturado algunas dinámicas del hábitat residencial local. Desde la perspectiva municipal, se trata de una falla. Desde una perspectiva económica, se trata de un accionar municipal perfectamente confluyente con las dinámicas de acumulación privada de riqueza urbana en forma de renta de suelo.

"A la única parte que hemos Ilegado con suficiente antelación ha sido al sector sur-poniente, al sector de Maestranza San Eugenio, a ese sector se llegó antes que el mercado con esta modificación del Plan Regulador [...] yo te digo que habían dos proyectos grandes ahí a punto de ser construidos, y [las inmobiliarias] ya habían comprado los terrenos, e iban a meter al medio de todas esas poblaciones obreras varios edificios así, tipo Paz Froimovich" (entrevista realizada a funcionaria del Departamento de Urbanismo del Municipio de Santiago, abril 2012).

La regulación ex post de la actividad inmobiliaria ha permitido su desplazamiento sin que el municipio direccione espacialmente posibles localizaciones, sino más bien mediante la aplicación de normas restrictivas en determinadas zonas ya saturadas de edificación en altura, o espacios necesarios de conservar por motivos sociales, políticos o por demandas patrimoniales o ambientales de la población residente. Luego, las zonas donde se relocaliza la actividad inmobiliaria son aquellas que todavía no han sido normadas, las cuales reciben el desarrollo en su máxima intensidad posible, hasta que una nueva modificación de Plan Regulador se haga por las razones anteriormente mencionadas.

En el caso de la comuna de Santiago, ha sido así la dinámica generada a través de las modificaciones denominadas "Sector Norponiente" (2003), "Triángulo Central" (2008), "Expansión del Centro" (2011) y "Club Hípico-Parque O’Higgins" (2012). Si bien no es claro que se trate de una estrategia de movilización de actividad inmobiliaria claramente planificada por parte del municipio, la fragmentación del territorio comunal a través de estas modificaciones parciales sí ha producido una secuencia de desplazamiento de la actividad inmobiliaria entre todas estas zonas de la comuna. Por ejemplo, la rápida explosividad en el mercado de Santa Isabel fue, antes que todo, producto de la protección normativa que redujo drásticamente la intensidad permitida del mercado en el sector norponiente de Santiago (Barrio Yungay) en 2003.

En cuanto al anclaje inicial de la renovación en la zona de Santa Isabel, esto obedece más bien a la aplicación de instrumentos de regulación bastante novedosos. Así, en 1995 aparece el "Seccional Santa Isabel", y luego en 1996 aparecen otros dos seccionales complementarios de Remodelación y de Zonas de Construcción Obligatoria para la misma zona $^{10}$, instrumentos existentes en la legislación urbanística chilena pero hasta entonces poco utilizados por gobiernos locales, haciendo de la autoridad municipal de Santiago un ente regulatorio de suelo particularmente funcional al desarrollo de proyectos detonantes a gran escala.

El primero de estos instrumentos, el "Seccional de Remodelación Santa Isabel", establecía condiciones normativas para un desarrollo morfológico "deseable" caracterizado por coeficientes mínimos de constructibilidad de 1,5 y 2,2 pero sobre extensas superficies de suelo a desarrollar (con mínimos exigidos de lote de $2.000 \mathrm{~m}^{2}$ y $5.000 \mathrm{~m}^{2}$ ). En otras palabras, se estableció una normativa que anulaba las posibilidades de conseguir permisos de edificación para obras de pequeña escala y capital, por otra parte incentivando la fusión predial hasta paños grandes, lejos del alcance de cualquier particular, y la posterior construcción inmobiliaria solo por parte de actores con alta capacidad de inversión, y evidentemente sofisticada capacidad técnica de construcción por el volumen buscado de los edificios. En concreto, se trata de un proceso de redlining institucional de la forma en que la define por ejemplo Aalbers (2007, 2011) para casos europeos y norteamericanos, y López Morales (2013a, 2013b) para casos chilenos (este concepto indica la delimitación deliberada de una zona urbana, con el fin de impedir el desarrollo de proyectos inmobiliarios fuera de las escalas deseadas

\footnotetext{
10 D.O. 24 de junio de 1996 Seccional de Remodelación de Santa Isabel y Seccional de Zonas de Construcción Obligatoria de Santa Isabel.
} 
Cuadro $\mathrm{N}^{\circ} 1$

Resumen de modificaciones aplicadas al Plan Regulador Comunal de Santiago

\begin{tabular}{|c|c|c|c|c|c|c|c|c|c|}
\hline \multirow[b]{2}{*}{ ZONAS } & \multicolumn{2}{|c|}{ 1990-1995 } & \multicolumn{3}{|c|}{ 1995-2006 } & \multicolumn{2}{|c|}{ 2006-2011 } & \multicolumn{2}{|c|}{2011} \\
\hline & B & B1 & B1a & B1b & B1c & $\mathrm{B}$ & B & B2a & B2a \\
\hline Superficie predial mínima (m) & \multirow{2}{*}{$\begin{array}{r}500 \\
1\end{array}$} & \multirow{2}{*}{$\begin{array}{r}1000 \\
0,8\end{array}$} & \multirow{2}{*}{$\begin{array}{r}5000 \\
0,8\end{array}$} & \multirow{2}{*}{$\begin{array}{r}2000 \\
0,8\end{array}$} & \multirow{2}{*}{$\begin{array}{l}500 \\
0,8\end{array}$} & \multirow{2}{*}{$\begin{array}{r}500 \\
1\end{array}$} & \multirow{2}{*}{$\begin{array}{l}500 \\
0,7\end{array}$} & \multirow{2}{*}{$\begin{array}{l}500 \\
0,7\end{array}$} & \multirow{2}{*}{$\begin{array}{l}500 \\
0,7\end{array}$} \\
\hline $\begin{array}{l}\text { Coeficiente máximo de } \\
\text { ocupación de suelo }\end{array}$ & & & & & & & & & \\
\hline Sistema de agrupamiento & \multirow{2}{*}{$\begin{array}{r}\text { A-P-C } \\
\text { N/A }\end{array}$} & \multirow{2}{*}{$\begin{array}{c}\text { A-P-C } \\
\text { N/A }\end{array}$} & \multirow{2}{*}{$\begin{array}{l}\text { A-P-C } \\
\text { N/A }\end{array}$} & \multirow{2}{*}{$\begin{array}{l}\text { A-P-C } \\
\text { N/A }\end{array}$} & A-P-C & A-P-C & A-P-C & A-P-C & \multirow{2}{*}{$\begin{array}{r}\text { A-P-C } \\
5,5 \\
(2)\end{array}$} \\
\hline $\begin{array}{l}\text { Altura mínima edificación } \\
\text { continua m (pisos) }\end{array}$ & & & & & N/A & N/A & N/A & $5,5(2)$ & \\
\hline $\begin{array}{l}\text { Altura máxima edificación } \\
\text { continua m (pisos) }\end{array}$ & \multirow{2}{*}{$\begin{array}{r}\text { 35(14) } \\
\text { sí }\end{array}$} & $35(14)$ & $35(14)$ & $35(14)$ & $35(14)$ & $35(14)$ & $22,5(9)$ & $32,5(13)$ & $\begin{array}{r}27,5 \\
(11)\end{array}$ \\
\hline Aislado sobre continuo & & \multirow{4}{*}{$\begin{array}{r}\text { sí } \\
1,5 \\
\text { N/A } \\
1 \mathrm{e} / 5 \mathrm{v}\end{array}$} & \multirow{4}{*}{$\begin{array}{r}\text { sí } \\
2,2 \\
\mathrm{~N} / \mathrm{A} \\
1 \mathrm{e} / 5 \mathrm{v}\end{array}$} & \multirow{4}{*}{$\begin{array}{r}\text { sí } \\
2,2 \\
\text { N/A } \\
1 \mathrm{e} / 5 \mathrm{v}\end{array}$} & \multirow{4}{*}{$\begin{array}{c}\text { no } \\
\mathrm{N} / \mathrm{A} \\
\mathrm{N} / \mathrm{A} \\
1 \mathrm{e} / 5 \mathrm{v}\end{array}$} & \multirow{4}{*}{$\begin{array}{r}\text { sí } \\
\mathrm{N} / \mathrm{A} \\
\mathrm{N} / \mathrm{A} \\
1 \mathrm{e} / 5 \mathrm{v}\end{array}$} & no & no & no \\
\hline $\begin{array}{l}\text { Coeficiente mínimo de } \\
\text { constructibilidad }\end{array}$ & \multirow{3}{*}{$\begin{array}{c}\mathrm{N} / \mathrm{A} \\
\mathrm{N} / \mathrm{A} \\
1 \mathrm{e} / 5 \mathrm{v}\end{array}$} & & & & & & $\mathrm{N} / \mathrm{A}$ & N/A & N/A \\
\hline $\begin{array}{l}\text { Coeficiente máximo de } \\
\text { constructibilidad }\end{array}$ & & & & & & & 3,6 & 5 & 4,3 \\
\hline Exigencia estacionamientos & & & & & & & $1 \mathrm{e} / 2 \mathrm{v}$ & $1 e / 2 v$ & $1 e / 2 v$ \\
\hline $\begin{array}{l}\text { Sistema de agrupamiento: } \mathrm{A}= \\
\text { trucciones que comparten un } \\
\text { partiendo ambos deslindes; y } \\
\text { que continua por sobre ella d }\end{array}$ & islado, & onctro & iones & 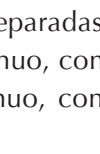 & de tod & loc do & lind & $=$ Pareac & cons- \\
\hline
\end{tabular}

Fuente: Elaboración propia (2013) en base a Ordenanzas Locales.

por la política municipal). En paralelo, el "Seccional de Remodelación" de Santa Isabel ofreció durante cinco años (hasta 2001) una exención de $80 \%$ del pago por concepto de derechos municipales de construcción, con lo que en la práctica se suprimió casi por completo el único mecanismo de captura municipal de recursos por esta actividad, a beneficio de los desarrolladores. Los derechos municipales de Construcción corresponden al 1,5\% del costo total de la obra, el cual ya es rebajado en Chile entre un $30 \%$ y $40 \%$, por la repetición de "pisos tipo" en edificios en altura (Artículo 131 de la Ley General de Urbanismo y Construcción) y en un 30\%, por presentar un revisor Independiente privado por parte de la inmobiliaria (Artículo 3.1.8. de la Ordenanza General de Urbanismo y Construcción), por lo que una exención del $80 \%$ en Santa Isabel, dejó un monto final bastante marginal a pagar al municipio.
El segundo de estos instrumentos, el "Seccional de Zonas de Construcción Obligatoria", impuso el pago de una contribución territorial adicional del $100 \%$, y progresiva hasta $200 \%$, a los dueños de los predios declarados por el municipio y el Servicio de Vivienda Urbanización (SERVIU) como eriazos y "ruinosos"11 y que no realicen inversión inmobiliaria. Sin embargo, al haber establecido el municipio anteriormente condiciones mínimas de renovación que solo podrían ser cumplidas por actores con gran capacidad económica y técnica, este seccional se convirtió en una presión normativa importante para que propietarios privados de escaso capital y sin capacidad económica ni técnica de construcción en altura, vendiesen rápidamen-

\footnotetext{
${ }^{11}$ Definido en esos términos en D.O. 24 de junio de 1996.
} 
Figura $N^{\circ} 1$

Catastro de intervenciones y Seccional de Construcción obligatoria en predios eriazos y "ruinosos"
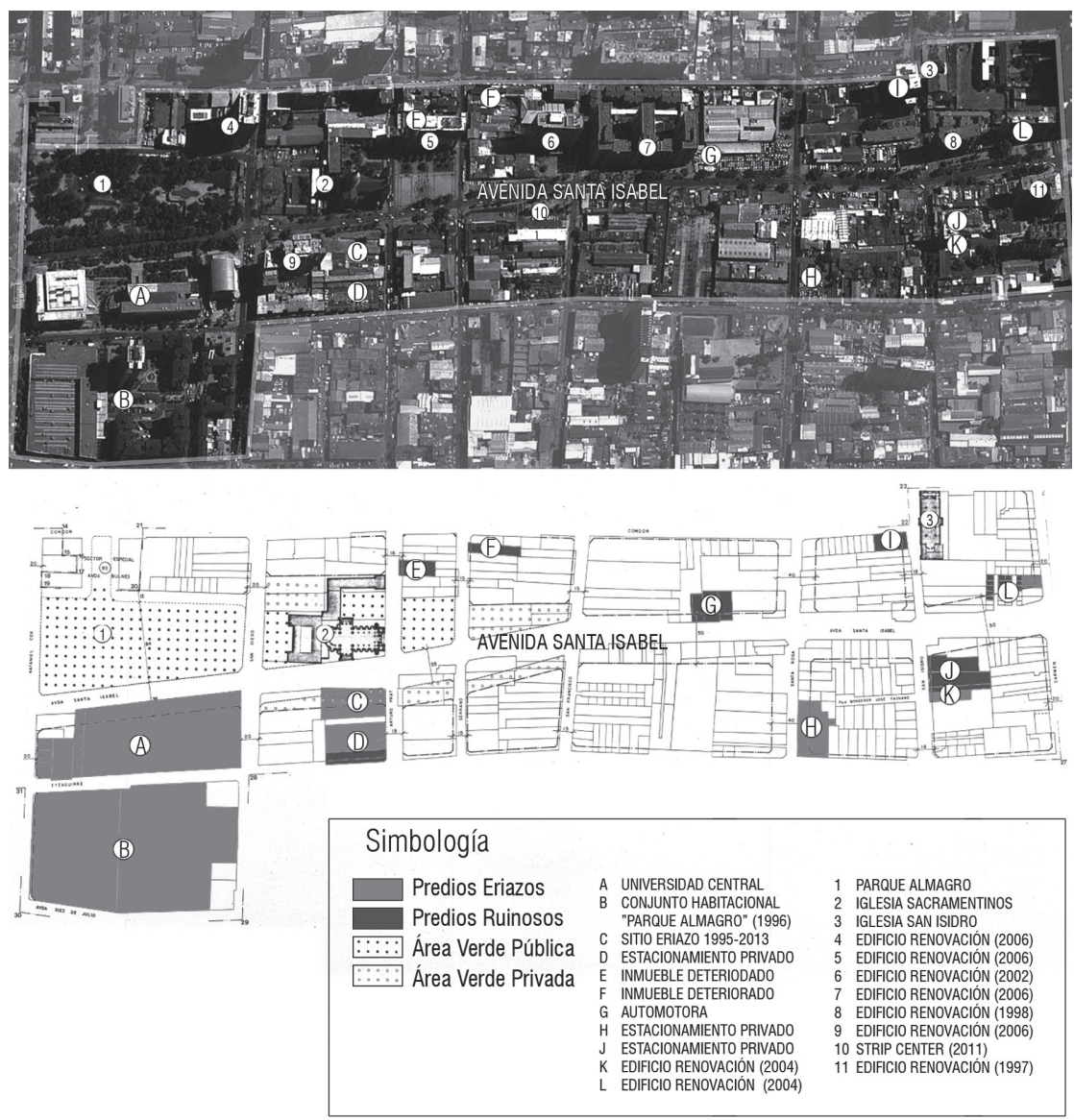

Fuente: Elaboración propia (2013) en base a foto Google Earth y plano DOM I. Municipalidad de Santiago.

te sus paños de suelo a los desarrolladores, cosa que finalmente ocurrió.

Por medio de estos instrumentos se generó el marco de acción orientado a anidar las primeras "macroinmobiliarias" que desarrollaron proyectos de construcción en el sector de Santa Isabel, tanto residenciales como orientados a la educación superior privada (Figura $\mathrm{N}^{\circ} 1$ ). Lo particular de esta intervención es que mediante la regulación urbanística de 1996 se establece una franja a lo largo del eje Santa Isabel, con condiciones mínimas y máximas de edificación definidas con alto grado de especificidad. En 1995, la capacidad productiva para construir, medida en términos cuantitativos, exhibida por las empresas, no era tan alta como la actual (por entonces, rondaba en los $4 \mathrm{mil} \mathrm{m}^{2}$ por proyecto); luego de la modificación de 1996, esa capacidad crecería a más de $15 \mathrm{mil} \mathrm{m}^{2}$ por proyecto. Como ilustra el Cuadro $\mathrm{N}^{\circ}$ 1, la zonificación B1a y B1b se orienta a la atracción de proyectos inmobiliarios de gran extensión, de los cuales fueron materializados solo tres: el campus de la Universidad Central $\left(87.000 \mathrm{~m}^{2}\right)$, y los conjuntos Parque Almagro $\left(65.000 \mathrm{~m}^{2}\right)$ y Parque Sacramentinos $\left(35.000 \mathrm{~m}^{2}\right)$.

Es en este punto cuando se marca una inflexión, incrementándose la capacidad constructiva del suelo en el sector Santa Isabel. Tanto las zonas para proyectos en densifica- 
ción extendida (zonas B1a y B1b), como las zonas que permitían un desarrollo de proyectos de densificación puntual (B1 y B1c) acogen proyectos de gran superficie construida, por sobre el promedio comunal. Particularmente los proyectos de esta última tipología, modalidad densificación puntual, por ejemplo " 11 " y " 8 " de la Figura $^{\circ} 1$, comienzan ya durante la segunda mitad de la década de 1990, a alcanzar el límite con la zonificación B1c del Seccional Santa Isabel, más "interior" y residencial en menor intensidad.

\section{Consolidación del mercado de renovación urbana con crecimiento de las utilidades por renta de suelo}

Durante el período de vigencia de la normativa del Seccional Santa Isabel (19952006) se registraron dos procesos que definirían la hiperactividad inmobiliaria posterior en el desarrollo del sector Santa Isabel, mediante proyectos "puntuales", es decir, de menor consumo de suelo pero de mayor densidad. El primero fue el inicio de un proceso de actualización del Plan Regulador, que subdividió el territorio de la comuna en seis zonas para realizar una movilización efectiva de la actividad inmobiliaria, desde zonas ya "saturadas", gravadas con normas restrictivas, hacia nuevas zonas "vírgenes" gravadas con normas permisivas (López Morales et al., 2012). El segundo fue la corporatización inmobiliaria luego de la emigración de las cooperativas abiertas de vivienda, que ya se mencionó más arriba, que marca un vuelco al modo actual de producción inmobiliaria de la renovación urbana.

En este marco es que se consolidaron cuatro inmobiliarias dominantes, a saber Paz-Froimovich, RVC, Euro y Absal, las que desarrollan proyectos más cercanos a la modalidad de densificación puntual en edificios de alta intensidad. Paz-Froimovich por años había estado presente en la mesa directiva de la CORDESAN como representante de la Cámara Chilena de la Construcción. Los cuatro conglomerados realizaron sus primeros proyectos en la zona Santa Isabel, para luego exportar su modelo de renovación en altura hacia otras comunas y regiones del país.
No obstante, el punto de inflexión que marcaría el desarrollo del sector Santa Isabel sería un nuevo ajuste normativo, más bien una omisión, para ser más precisos. Ya en 1996, el término del beneficio de exención de derechos municipales, el agotamiento del banco de suelo de la CORDESAN, y la dificultad para generar banco de suelo privado de gran extensión, generaba muchas restricciones para la consolidación del proceso de renovación ya alcanzado. De esta forma, en la actualización del texto de la normativa comunal de 2006 (D.O. 12 de enero 2006), se refundió la ordenanza para incorporar todas las zonificaciones comunales correspondientes a los planes seccionales realizados durante la década pasada, salvo específicamente los seccionales de Santa Isabel, que fueron eliminados. ¿Por qué se hizo esto? Sostenemos que porque esta derogación fue clave al volver a instaurar la zonificación y normativa de 1990, netamente permisiva, abriendo las posibilidades de continuar desarrollando una densificación intensificada en paños puntuales por toda la zona Santa Isabel. La derogación de 2006 traería un alza importante en la cantidad de proyectos desarrollados en la zona, así como un incremento notable en el volumen de renta de suelo capturada por inmobiliarias ${ }^{12}$. La Figura $\mathrm{N}^{\circ} 2$ muestra el incremento y "peak" en 2007 del total de la renta de suelo anual capturada por inmobiliarias (color negro), "extraída" de la zona, durante el periodo 2000-2012.

\footnotetext{
12 Las utilidades privadas por renta de suelo, o brecha de renta, se calculan como la ganancia obtenida por el productor inmobiliario por unidad de superficie de suelo, descontando todos los costos asociados a la ejecución del proyecto. En clave de fórmula: $\mathrm{Br}=[\mathrm{V}-$ $\mathrm{S}-\mathrm{C}$ ] / Sup, donde $\mathrm{V}$ corresponde al valor de venta de todas las unidades residenciales del edificio ofertadas, $\mathrm{S}$ es el precio de suelo pagado para construir (de acuerdo a la información del CBRS), C los costos de construcción (valor unitario entregado por el Ministerio de Vivienda y Urbanismo, multiplicado por la cantidad de metros cuadrados construidos) y Sup la superficie del predio desarrollado, en metros cuadrados. Se plantean dos supuestos para el cálculo: que todos los departamentos son vendidos por proyecto, y se estima su rentabilidad en un periodo anual sin cálculo de valor actualizado neto (VAN) para años sucesivos. Ambos supuestos son posibles en un mercado con velocidades de venta generalmente altas. Se trata de un cálculo de brecha de renta tendencial y con fines comparativos de los 67 proyectos construidos en Santa Isabel en el periodo 2000-2010.
} 
Por su parte, es posible observar un aumento significativo de las brechas de renta en el período estudiado, sobre todo después de 2006, tras la derogación de los seccionales. Los 67 proyectos inmobiliarios analizados dentro del sector Santa Isabel en total consumieron 180 predios (posteriormente fusionados) cuyo precio pagado a sus antiguos dueños también fue observado a través de una indagación exhaustiva en los registros del Conservador de Bienes Raíces de Santiago. Se analizaron también los precios promedio de la oferta inmobiliaria para las distintas tipologías contenidas en cada edificio, en plataformas digitales especializadas (portal inmobiliario.com). De esta manera se analizaron las rentas obtenidas por los agentes económicos en las zonas de estudio, identificando para cada proyecto la ganancia neta por unidad de superficie capitalizada por la empresa inmobiliaria descontado el pago por suelo y costos de producción, y la renta obtenida por los propietarios de suelo que venden predios al mercado de renovación (sumatoria de las rentas de suelo obtenidas por estos agentes en un mismo proyecto). La Figura $N^{\circ} 2$ muestra datos agregados en tres subperíodos. Se observa que entre 2001-2004 las utilidades estaban cerca de las $46 \mathrm{UF} / \mathrm{m}^{2}$, mientras en el subperíodo 2009-2012 se acercaron a 94 UF/ $\mathrm{m}^{2}$, representando un aumento del $103 \%$.

Por su parte, la misma figura muestra que las rentas obtenidas por los propietarios antiguos de los predios desarrollados aumentó, entre los subperíodos 2001-2004 y 20092012, solo en un $20 \%$, Ilegando en promedio a una cifra levemente inferior a $10 \mathrm{UF} / \mathrm{m}^{2}$. Consideramos que está altamente correlacionado con la situación monopsónica de compra de suelo, y la altísima disparidad de poder de negociación del precio de compra/venta exhibida por los actores desarrolladores y propietarios respectivamente. Los datos revelan una situación de altísimo incremento del diferencial entre nivel de captura de renta de sue-

Figura $N^{\circ} 2$

Gráficos de comparación entre utilidades inmobiliarias y precios pagados por suelo a propietarios en zona Santa Isabel (2000-2012). Valores globales (izquierda, en dos escalas) y relativos en $U F / m^{2}$ (derecha)

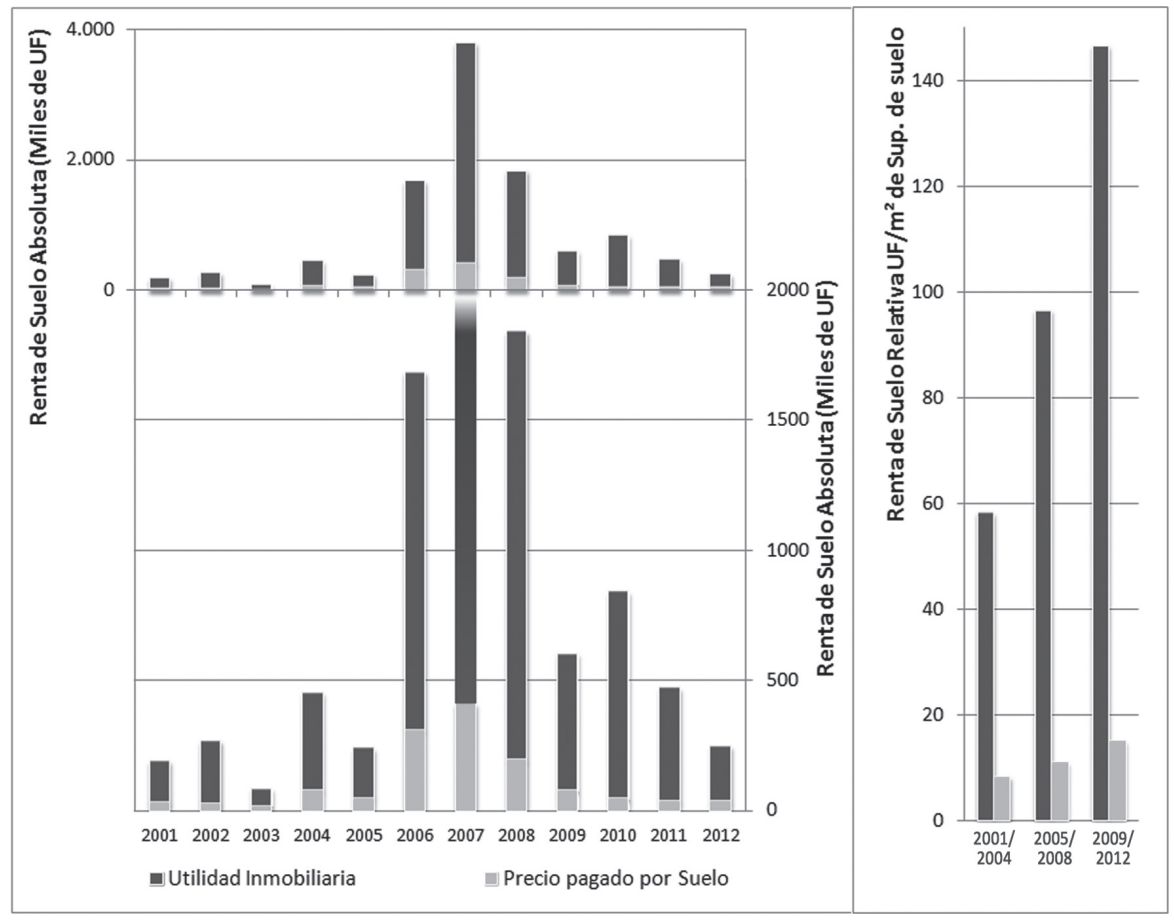

Fuente: Elaboración propia. 
lo por inmobiliarias y propietarios particulares en el período estudiado, donde la ganancia corporativa en gran medida se sustenta en los escasos niveles de renta de suelo percibida por los propietarios, ligado al aumento derivado de la mayor extracción de utilidades por parte del productor inmobiliario.

Sin embargo, este periodo de intenso desarrollo inmobiliario en Santa Isabel decaería con el estancamiento del sector inmobiliario durante la crisis de 2009, el terremoto de 2010 $y$, principalmente, el congelamiento de permisos de edificación entre 2010 y 2011 requerido para realizar un último ajuste normativo como parte del ya mencionado proceso de actualización del Plan Regulador del año 2011. En este caso, la llamada "Modificación 3 y 4, Barrio Universitario y Expansión del Centro" fue aprobada en septiembre de 2011, con lo cual se estableció límites definidos para la edificación en altura (Cuadro $\mathrm{N}^{\circ}$ 1). Si bien esta nueva modificación tampoco considera el establecimiento de densidad máxima, lo que sí generó fue un gran incentivo a la oferta de departamentos más amplios, disminuyendo la cantidad de unidades por proyecto y por ende la altísima densidad residencial que se había generado en la zona en el decenio anterior. La modificación de 2011 también limitó las condiciones morfológicas que los proyectos podrían adoptar, estableciendo como altura máxima los 13 pisos, y como coeficiente de constructibilidad máximo un factor de 5, respectivamente. Dicha normativa aún se considera como reciente, por lo que todavía no se observan variaciones importantes, aunque sí permite predecir que el boom inmobiliario de Santa Isabel seguirá en descenso, desplazándose nuevamente la inversión privada a las zonas al sur de la comuna de Santiago (sector Matta-Franklin), con valores potenciales de renta de suelo comparativamente más atractivos para el desarrollador.

\section{Gentrificación en la forma de "desplazamiento exclusionario" en Santa Isabel}

Eric Clark, en un capítulo de libro publicado en 2005 denominado "El orden y simplicidad de la gentrificación", definió genéricamente la gentrificación como el desplazamiento de usuarios de bajos ingresos, desde un sector urbano, por otros usuarios nuevos de mayores ingresos, en un contexto de comodificación del espacio y relaciones de poder polarizadas. La definición de Clark ha sido altamente influyente, y se relaciona con el concepto de "desplazamiento exclusionario" definido por Marcuse (1985) y luego por Slater (2009), concepto que López Morales (2013b) interpreta para la realidad chilena (basada en altas tasas de propiedad "social" de suelo urbano que caracteriza originalmente a la mayoría de las zonas de renovación urbana en el país) como la imposibilidad o dificultad de los propietarios-residentes que han vendido sus predios al mercado de la renovación urbana, de adquirir suficiente compensación por suelo, ergo poder relocalizarse, en viviendas nuevas ofertadas dentro de la misma zona. La hipótesis que sostiene este apartado es la siguiente: la gentrificación en el centro y pericentro de Santiago es un proceso de remercantilización del suelo urbano por ingreso de capital inmobiliario de renovación urbana que comporta apropiación desigual de renta urbana con efecto de desplazamiento exclusionario de los residentes originales de las áreas centrales. En términos estrictamente de la economía política, son las inmobiliarias las que producen la gentrificación, y no los nuevos usuarios que llegan a residir en sus productos y que, para efectos de las transformaciones en las economías locales de suelo, son factores prácticamente irrelevantes (López Morales, 2013a, 2013b). Es necesario por ende observar la relación existente entre el valor capturado, en forma de renta, por los hogares propietarios-residentes que venden sus predios a proyectos de renovación y los precios de las viviendas ofertadas para su relocalización residencial en el lugar.

También es necesario caracterizar los hogares con los cuales se trabajó, para comprender aspectos generales del proceso social. En 2012 levantamos en Santiago una encuesta a un total de 746 hogares residentes en lotes próximos a zonas de desarrollo urbano en Santiago. En el caso del sector Santa Isabel, fueron 196 hogares encuestados. Según los datos recabados, la distribución por sexo y edades de la población residente antigua de los barrios sometidos a renovación es diferente al promedio de la población urbana chilena. En promedio, el segmento de 20 a 34 años está sobrerrepresentado, además de mostrar una significativa presencia de adultos mayo- 
res, particularmente de sexo femenino (53\%). La zona Santa Isabel muestra una relativamente baja escolaridad y predominan jefes de hogar que solo cursaron hasta enseñanza media. Ante la pregunta sobre ingresos mensuales por hogar, aparece que en un $54 \%$ de los casos los valores son inferiores a $\$ 600.000(2,86$ sueldos mínimos), reflejando así la vulnerabilidad socioeconómica de gran parte de la población original residente, cuyo único capital es el suelo que ocupan.

Ahora bien, para observar la relación entre la renta del suelo capturada por los hogares propietarios y los precios de las viviendas de relocalización en el lugar, realizamos un análisis de "tasa de relocalización" que estima estadísticamente el porcentaje del precio de vivienda nueva de reemplazo que tendrían los propietarios de suelo si vendiesen su propiedad y ocuparan el total de lo obtenido, con el fin de relocalizar su grupo familiar en una vivienda nueva dentro de la misma zona. Se trata evidentemente de un supuesto analítico. En base a la encuesta a residentes realizada en 2012, se plantearon tres escenarios estadísticamente probables de captura de renta de suelo (bajo, medio y alto), debido a la considerable desviación estándar en los precios pagados por suelo observada entre 2000 y 2010 . El escenario de "baja renta", que es una media del tercio de rentas más bajas, corresponde a un valor de $7,5 \mathrm{UF} / \mathrm{m}^{2}$. El 'escenario de "alta renta", agrupa el tercio de rentas más altas con un valor medio de 21,2 UF/m². Por último, el escenario de "renta media" corresponde al valor promedio de las rentas obtenidas es igual a $14,7 \mathrm{UF} / \mathrm{m}^{2}$. Se estratificaron también los precios de las tipologías residenciales nuevas ofertadas en la misma zona, requeridas por los propietarios-residentes para relocalizarse, ordenando todo el stock nuevo existente entre 2006 y 2010, desde los precios de venta más bajos a los más altos. Luego se imputaron valores de pago por suelo (según cada escenario) de acuerdo al tamaño de cada lote de suelo encuestado. Se correlacionó el tamaño del hogar con la tipología de vivienda nueva necesaria para cada núcleo familiar. Más antecedentes en López Morales et al. (2013).

Los resultados obtenidos para la zona de Santa Isabel son particularmente bajos, si se les compara con otras zonas del pericentro del Gran Santiago. En esta zona existe una cantidad importante de predios pequeños, por debajo de los $100 \mathrm{~m}^{2}$ incluso, que reportarían rentas de suelo muy bajas a los propietarios que vendan. A esto se suma que no existe relación inversamente proporcional entre renta de suelo y superficie del predio, sino que la renta se mantiene constante y la superficie del paño no es factor explicativo o determinante en el precio de la propiedad. Esto indica que es poco verosímil la consideración de que los predios más pequeños se puedan vender a mayor precio unitario de suelo, lo que refuerza la idea de que en Santa Isabel exista mayor probabilidad de desplazamiento de los residentes, respecto a otras áreas centrales de renovación urbana de Santiago.

72 hogares encuestados en Santa Isabel son propietarios, y en base a la superficie predial de cada una de sus propiedades ocupadas, 16 no tienen posibilidad alguna de acceder al mercado por integrar familias de 5 o más habitantes. La inexistencia de oferta de más de 3 dormitorios imposibilita o dificulta sustantivamente en la práctica la relocalización de dichas familias en las viviendas de renovación. O posibilita esa relocalización pero en condiciones de alto hacinamiento. Esta situación debe ser entendida como un primer tipo de exclusión socioespacial por no cobertura de la demanda, y puede ser progresivamente mayor su relevancia de estudio si se considera que la oferta de departamentos de 3 dormitorios viene reduciéndose sostenidamente en el más reciente período 2010 2013. Según datos de Portal Inmobiliario, la oferta de departamentos de 3 dormitorios a inicios de 2010 representaba el 17\% del total ofertado, comportando una baja sostenida, hasta representar menos del $10 \%$, a partir de fines del 2011. Este problema de hacinamiento potencial postrelocalización también se aplica a hogares de dos o tres personas, ante la expectativa de ocupar departamentos de un solo ambiente, que representan el $45 \%$ de la oferta residencial nueva en la zona.

Luego, existe un grupo de propietarios que comporta exclusión absoluta del mercado en tanto las rentas que pueden obtener no permiten solventar la compra de ninguna vivienda de renovación. Al estratificar los precios de todos los departamentos ofertados, 
se observa que las rentas de suelo a obtener por estos propietarios son inferiores al precio mínimo del departamento de la tipología que requieren.

En la Figura $N^{\circ} 3$ se observan tasas de relocalización dentro de un "escenario promedio" de pago por compra/venta de suelo. Los resultados muestran que un $17,5 \%$ de los propietarios-residentes encuestados en Santa Isabel tienen tasas inferiores a 0.5. Esto indica que, en un escenario de pago promedio por paño de suelo y según la superficie predial de cada propietario, la renta a obtener alcanza- ría para costear menos de la mitad del precio del departamento que requiere el hogar propietario en la zona, de acuerdo a su cantidad de integrantes. Y solo un $49 \%$ de los hogares residentes alcanzarían a solventar el precio del departamento requerido con la renta de suelo obtenida tras la venta de su propiedad actual. Si se observa el escenario bajo, se obtiene que un $34 \%$ de los propietariosresidentes en Santa Isabel no tiene capacidad de solventar el departamento más barato ofertado con el valor obtenido por la venta de su propiedad.

Tasas de relocalización de propietarios-residentes en Santa Isabel

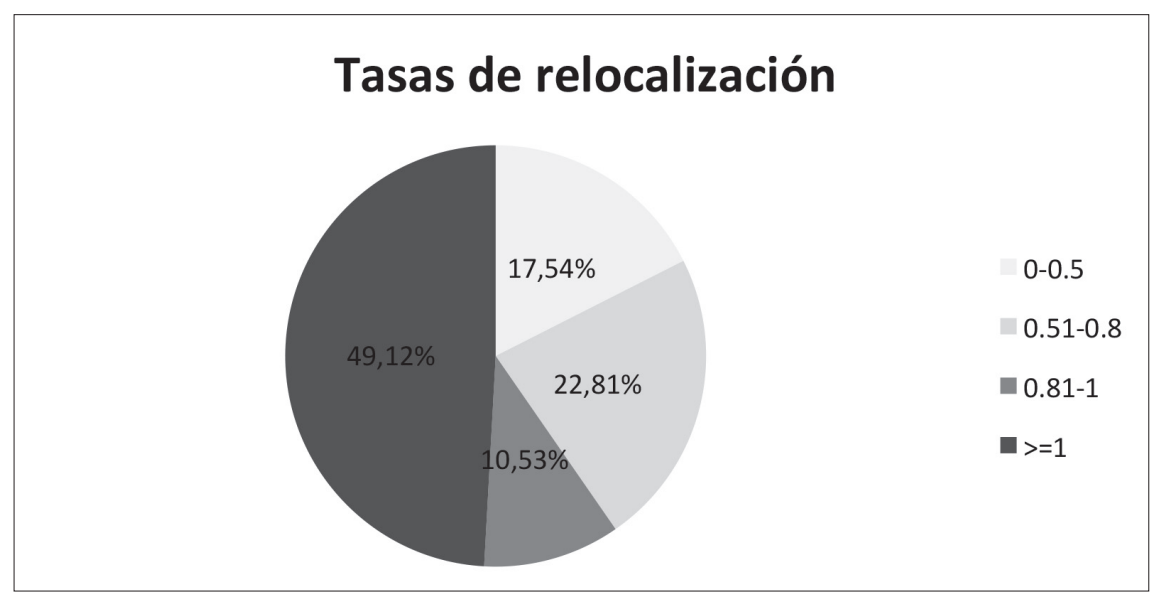

Fuente: Elaboración propia.

Luego es importante visualizar cómo varía este grupo de exclusión de acuerdo a los tres escenarios mencionados. En el "escenario de baja renta", es decir, capturando una renta de $7,5 \mathrm{UF} / \mathrm{m}^{2}, 33$ de los 56 hogares presentan exclusión absoluta. Este grupo se reduce radicalmente a dos hogares cuando se plantea el 'escenario de alta renta' de suelo. Por su parte, el 'escenario medio' indica un total de 11 hogares encuestados que no podrían relocalizarse incluso en las viviendas más baratas ofertadas. Es decir, un 20\% de los casos.

Por su parte, se observan otros resultados interesantes cuando se analiza la exclusión según porcentajes de la oferta de vivienda producida en la zona. Así, en el "escenario medio" habría 19 de los 56 hogares que no estarían en condiciones de relocalizarse en el $90 \%$ de las viviendas nuevas ofertadas. Es un grupo social que tiene posibilidad de relocalizarse en un $10 \%$ o menos del stock producido, lo cual refleja escasa capacidad de elección y un alto grado de exclusión del mercado. Para el "escenario de baja renta", el grupo de residentes excluidos del $90 \%$ del stock de vivienda asciende a 38 hogares, y para el "escenario de alta renta" se mantendría en una cantidad muy baja de 3 hogares. En rigor, el "escenario de alta renta" presenta recién niveles significativos cuando se observa la exclusión del 50\% del mercado, con un total de 12 hogares. La pregunta acerca de hacia dónde se han estado movilizando 
Figura $\mathrm{N}^{\circ} 4$

Stock de relocalización según escenarios de alta, media y baja renta en Santa Isabel

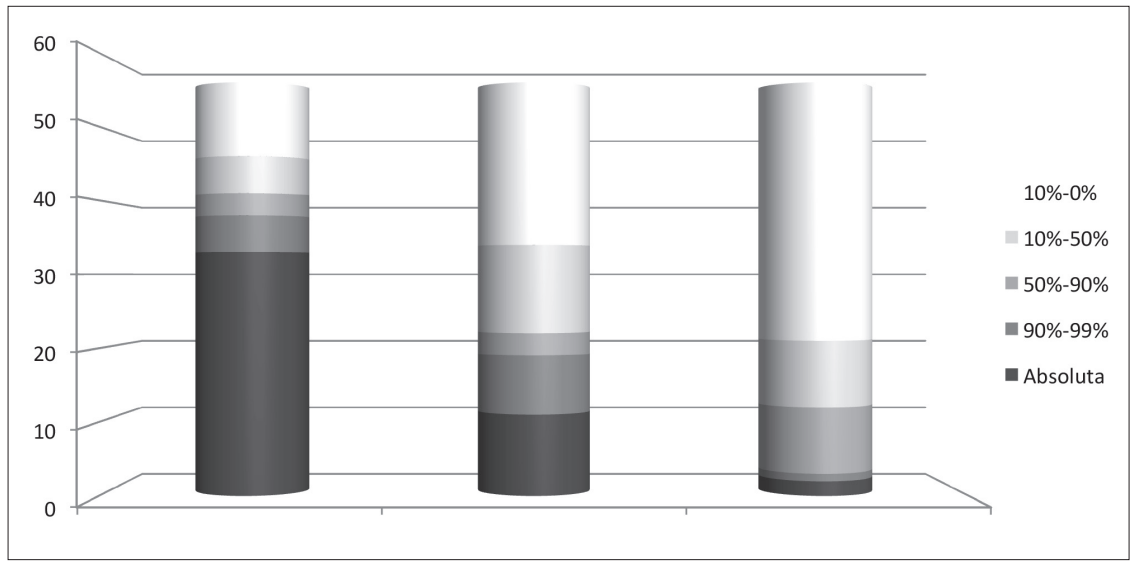

Fuente: Elaboración propia (2013).

los hogares desplazados en las últimas dos décadas sigue abierta, y esperamos abordarla en investigaciones sucesivas para confirmar la hipótesis de que han sido direccionados a zonas de menores centralidad y calidad ambiental.

\section{Conclusiones}

Santa Isabel ha sido una zona urbana donde el Estado chileno ha intervenido deliberadamente para fijar condiciones normativas, selectivamente orientadas a promover cierta escala de producción y volumen de capital inmobiliario. La evidencia muestra una serie de decisiones políticas e implementaciones normativas de suelo y de proyectos detonantes, aplicadas tan atrás como desde 1985 (CORDESAN), 1991 (Subsidio de Renovación Urbana) y 1996 (Seccional Santa Isabel). De alguna forma se va produciendo una creciente articulación entre los aparatos municipal y sectorial nacional, no exenta de prueba/error, con el fin de intensificar redesarrollo en la zona. La inusual aplicación de tres seccionales en 1996 prepararon el terreno para la implantación a gran escala de inversión inmobiliaria, que logra incrementar el nivel de captura de renta de suelo en más de $100 \%$ en 12 años. Ello, en desmedro de la pequeña inversión en construcción, que fuera prohibida en el lugar hasta 2005, cuando ya no existía competencia posible para las bien afianzadas empresas inmobiliarias privadas, dominadoras de este nicho de mercado residencial.

Las sucesivas disposiciones normativas aplicadas en Santa Isabel, que posibilitan extender las utilidades de las empresas inmobiliarias por captura de renta de suelo, son evidencia del éxito del régimen pro empresarial vigente en Chile por más tiempo del que a menudo se le supone. Estas mismas normativas liberalizadoras de suelo se vuelven, en fases posteriores, un obstáculo para el desarrollo inmobiliario más intensivo, haciendo necesario el permanente ajuste ad-hoc de los instrumentos. Por otra parte, observamos que las rentas capturadas por los pequeños propietarios que venden suelo al mercado, casi no varían, manteniéndose en niveles regularmente bajos en la totalidad del proceso. Esto se transforma en un hallazgo preocupante, en la medida de que contradice el sentido común imperante de que "a mayor demanda, mayor precio del suelo". En rigor, lo que hay son una serie de contextos de compra de suelo por parte de inmobiliarias, con una alta disparidad en la capacidad de negociación entre vecinos residentes y compradores empresariales.

Los análisis de relocalización de residentes permiten evaluar el proceso de desplazamiento exclusionario en Santa Isabel. Que 
un $49 \%$ de los propietarios encuestados no alcance a costear mínimamente una vivienda de relocalización in situ con la renta obtenida por la venta de su predio, y que un $32 \%$ no se pueda relocalizar en el $90 \%$ de los departamentos ofertados en un escenario de renta media, son ambos antecedente tremendamente relevantes a considerar por la política pública, si lo que interesa es mejorar la condición de exclusión del mercado a la que se ven enfrentados los antiguos residentes de los principales barrios en renovación intensificada que hay actualmente en las ciudades metropolitanas de Chile.

Lo que ha experimentado la zona Santa Isabel es un proceso de gentrificación, a escala metropolitana, con un encarecimiento sostenido del precio de la vivienda nueva y niveles altos de desplazamiento socioespacial. Como en toda historia de gentrificación, hay desplazados y desplazantes. Los primeros son el $50 \%$ de los residentes locales. Los segundos son las inmobiliarias que operan en el sector, y que están incorporando residentes de segmentos medios con alto endeudamiento bancario. El problema radica en las condiciones en que ello ocurre, y si es éticamente justo aceptar tal nivel de desplazamiento social en un contexto de mercados inmobiliarios que generan tanta oferta residencial junto con tan altas utilidades corporativas por renta de suelo. Sería preocupante que los resultados actualmente exhibidos hayan correspondido a la imagen objetivo de renovación urbana que se definió a fines de la década de 1980.

También existe en Chile una alta necesidad de medir e internalizar externalidades del desarrollo urbano derivadas de la apropiación de plusvalías generadas por obras públicas, y especialmente, por los cambios intencionados de normas de construcción generados por los municipios que componen el área central y pericentral de Santiago. Sin estos profundos ajustes, será poco probable que el Estado chileno cuente con suficientes recursos financieros y políticos para lograr reproducir una real mixtura social en el centro y pericentro, y mantener y localizar residentes de bajos ingresos en estas zonas, respetando su derecho a la ciudad. Por la magnitud de la evidencia encontrada, pensamos que este caso de gentrificación del centro y pericentro de Santiago cobra una relevancia especial como ejemplo del desarrollo urbano actual altamente polarizado y excluyente, en las ciudades chilenas y latinoamericanas.

\section{Referencias bibliográficas}

AALBERS, M. The Financialization of Home and the Mortgage Market Crisis. Competition \& Change, 2007, Vol. 12, N ${ }^{\circ} 2, \mathrm{P}$. 148-166.

AALBERS, M. Place, exclusion, and mortgagemarkets. London: Blackwell Publishing, 2011.

ARRIAGADA, C.; MORENO, J.C., y CARTIER, E. Evaluación de Impacto del Subsidio de Renovación Urbana: Estudio del Área Metropolitana del Gran Santiago 1991-2006, Santiago de Chile: MINVU, 2007.

CATTANEO, R. Los fondos de inversión inmobiliaria y la producción privada de vivienda en Santiago de Chile. ¿Un nuevo paso hacia la financiarización de la ciudad? EURE, 2011, Vol. 37, No 112, p. 5-22.

CLARK, E. The order and simplicity of gentrification - a political challenge. In: ATKINSON, R. \& BRIDGE, G. (editors). Gentrification in a Global Context: The new urban colonialism. Oxon: Routledge, 2005, p. 256264.

CONTRERAS, Y. La recuperación urbana y residencial del centro de Santiago: Nuevos habitantes, cambios socio-espaciales significativos. EURE, 2011, Vol. 37, No 112, p. 89-113.

DELGADILLO, V. Mejoramiento habitacional en las áreas urbanas centrales de América Latina. Del combate de tugurios a la rehabilitación habitacional progresiva. Revista INVI, 2008, No 63, Vol. 23, p. 89-119.

DE MATTOS, C. y FUENTES, L. Crecimiento de la población de Santiago entre 2002 y 2012: ¿compactación o expansión? Una falsa disyuntiva. Planeo, 2012, № 8, $\mathrm{s} / \mathrm{p}$. Disponible en internet: revistaplaneo. uc.cl/2012/11/05/crecimiento-de-la-pobla- 
cion-de-santiago-entre-2002-y-2012-compactacion-o-expansion-una-falsa-disyuntiva

HERZER, H. Con el corazón mirando al sur. Transformaciones en el sur de la ciudad de Buenos Aires. Buenos Aires: Espacio Editorial, 2008.

JANOSCHKA, M.; SEQUERA, J. \& SALINAS, L. Gentrification in Spain and LatinAmerica - a Critical Dialogue. International Journal of Urban and Regional Research, 2014, Vol. 38, No 4, p. 1234-1265.

LÓPEZ MORALES, E. Urbanismo pro empresarial y destrucción creativa: un estudio de caso de la estrategia de renovación urbana en el pericentro de Santiago de Chile, 19902005, México: Infonavit-Redalyc, $2013 a$.

LÓPEZ MORALES, E. Gentrificación en Chile: aportes conceptuales y evidencias para una discusión necesaria. Revista de Geografía Norte Grande, 2013b, N 56, p. 31-52.

LÓPEZ MORALES, E.; GASIC, I. y MEZA, D. Urbanismo Pro-Empresarial en Chile: políticas y planificación de la producción residencial en altura en el pericentro del Gran Santiago. Revista INVI, 2012, Vol.
28, $N^{\circ}$ 76. Disponible en Internet: http:// www.scielo.cl/scielo.php?pid=S0718$83582012000300003 \&$ script=sci_arttext

MARCUSE, P. Gentrification, Abandonment, and displacement: connections, causes, and policy responses in New York City. Journal of Urban and Contemporary Law, 1985, $N^{\circ} 28$, p. 195-240.

ROJAS, E. Volver al centro. La recuperación de áreas urbanas centrales. Washington D.C: Banco Interamericano del Desarrollo, 2004.

SLATER, T. Missing Marcuse: On gentrification and displacement. City - Analysis of Urban Trends Culture Theory Policy and Action, 2009, Vol. 13, N², p. 292-312.

VALENZUELA, M. Programa de repoblamiento comuna de Santiago: Un programa de gestión urbana. Urbano, 2003, Vol. 6, № 8, p. 53-61.

ZUNINO, H. Power Relations in Urban Decision-making: Neo-liberalism, 'Technopoliticians' and Authoritarian Redevelopment in Santiago, Chile. Urban Studies, 2006, Vol. $43, N^{\circ} 10$, p. 1825-1846. 
